FRI0014

A PUTATIVE ROLE OF IGF-1R ON THE PATHOGENESIS OF GOUT THROUGH BINDING TO TRANSCRIPTION FACTORS

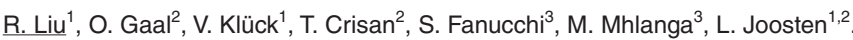
${ }^{1}$ Radboudumc, Nijmegen, Netherlands; ${ }^{2}$ "Iuliu Hatieganu"University of Medicine and Pharmacy, Cluj Napoca, Romania; ${ }^{3}$ Faculty of Health Sciences, University of Cape Town, Cape Town, South Africa

Background: Recent studies showed that SNPs on IGF-1/IGF-1R were highly associated with hyperuricemia and gout $[1,2]$. It was shown that the IGF-1/IGF-1R signaling pathway played a role in regulating the serum urate level. By modulating the uric acid transporters, IGF-1/IGF-1R influenced the resorption and secretion of uric acid. However, we demonstrated that the increased activation of IGF1R could activate the mTOR pathway, leading to a higher inflammatory response upon pathogen stimulation [3]. This finding indicates that IGF-1/IGF1-R has a role in inflammation, which could results in gout. The IGF-1/IGF-1R pathway may have an overall influence on both urate transporters and inflammatory pathways. it was shown that IGF-1R was not only expressed on the cell surface, but could also internalize into the nucleus and recruit RNA polymerase, regulating the expression of other transcription factors[4]. These transcription factors have been shown to regulate inflammation and have been predicted to bind promoter regions of urate transporters [5]

Objectives: To unveil how the IGF-1/IGF1-R associates with hyperuricemia and gout by studying the IGF-1R SNP rs6598541.

Methods: To assess the influence of the SNP to IGF1-R, the protein expression of IGF-1R on the cell surface was identified by flow cytometry in different genotypes. Additionally, we measured the in vitro immune response of PBMCs with different genotypes upon exposure to MSU and/or LPS. To estimate the overall influence of the SNP on the immune response, we analyzed the SNP's function on transcription factors.

Results: We observed an enhanced inflammatory response in the homozygous genotype with the risk alleles upon LPS and/or MSU stimulation, indicative of a higher risk for gout. However, the IGF-1R surface expression level was comparable between different genotypes. Furthermore, in epigenetic analysis, we found that rs 6598541 located in an enhancer region, which is bound by c-FOS, c-JUN and other transcription factors. In recent years, c-FOS and c-JUN have been shown to regulate inflammatory responses.

Conclusion: The risk allele of rs6598541 is associated with a higher inflammatory response, which might be the key factor for gout. Because of the location of the SNP, it might explain the function of IGF-1R in gout, and the pathogenesis might be modulated through transcription factors. According to the recent study, intracellular IGF-1R could act as a transcription factor regulating other transcription factors expression, like c-JUN. Additionally, C-JUN has been shown to regulate inflammatory responses. It is tempting to speculate that IGF-1R regulates transcription factors expression and leads to an overall immune responses, which influence the risk of gout.

References:

[1] Kottgen, A., et al., Genome-wide association analyses identify 18 new loci associated with serum urate concentrations. Nat Genet, 2013. 45(2): p. 145-54.

[2] Mannino, G.C., et al., The polymorphism rs35767 at IGF1 locus is associated with serum urate levels. Sci Rep, 2018. 8(1): p. 12255.

[3] Bekkering, S., et al., Metabolic Induction of Trained Immunity through the Mevalonate Pathway. Cell, 2018. 172(1-2): p. 135-146.e9.

[4] Aleksic, T., et al., Nuclear IGF1R Interacts with Regulatory Regions of Chromatin to Promote RNA Polymerase II Recruitment and Gene Expression Associated with Advanced Tumor Stage. Cancer Res, 2018. 78(13): p. 3497-3509.

[5] Granet, C., W. Maslinski, and P. Miossec, Increased AP-1 and NF-kappaB activation and recruitment with the combination of the proinflammatory cytokines IL-1 beta, tumor necrosis factor alpha and IL-17 in rheumatoid synoviocytes. Arthritis Res Ther, 2004. 6(3): p. R190-8.

Disclosure of Interests: Ruiqi Liu: None declared, Orsi Gaal: None declared, Viola Klück: None declared, Tania Crisan: None declared, Stephanie Fanucchi: None declared, Musa Mhlanga: None declared, Leo Joosten Consultant of: SAB member of Olatec Therapeutics LLC

DOI: 10.1136/annrheumdis-2020-eular.6679

\section{FRI0015 PHENOTYPE AND FUNCTION OF THE PERIPHERAL BLOOD DENDRITIC CELLS OF PSORIASIS PATIENTS WITH AND WITHOUT ARTHRITIS}

S. Schnitte ${ }^{1}$, A. Fuchs' ${ }^{1}$, T. Funk ${ }^{1}$, A. C. Pecher ${ }^{1}$, D. Dörfel ${ }^{1,2}$, J. Henes $^{1}$ ${ }^{1}$ University Hospital Tübingen, Internal Medicine II, Tübingen, Germany; ${ }^{2} \mathrm{KRH}$ Klinikum Siloah, Hannover, Germany
Background: Psoriasis is a frequent skin disease that can appear with an arthritic manifestation in approximately $30 \%$ of the cases [1]. The underlying excessive immune reaction caused by pro-inflammatory cytokines can be triggered by several risk factors [2]. Various subgroups of Dendritic cells (DCs) in the skin play a crucial role in the induction of the dermal inflammatory response [3]. Objectives: As the role of peripheral blood DCs remains unknown and the cause of an arthritic manifestation is still not completely understood [4], this project aimed to detect differences in phenotype or function of peripheral blood DCs in psoriatic patients with or without arthritis.

Methods: We analyzed peripheral blood cells of 60 psoriasis patients with and without arthritis. Different DC subpopulations were detected by flow cytometry. Monocyte-derived DCs were cultured with or without Lipopolysaccharides to gain immature (iDC) and mature $(\mathrm{mDC})$ cells. The $\mathrm{DC}$ phenotype was determined by staining with CD80, CD83, CD86, CD206, CCR7, CD1a HLA-DR, CD40, GPN-MB, DC209 and CD14. Their T-cell stimulatory capability was analyzed by co-incubation with Carboxyfluorescein succinimidyl ester stained lymphocytes and the quantification of CD4+ T-lymphocytes afterwards. To measure the migration capacity DCs were seated into transwell chambers with a semipermeable membrane and partly supplemented with Macrophage Inflammatory Protein 3 Beta (Mip3b). Migrated cells were detected by flow cytometry. Measured cell counts were normalized to cell counts without Mip3b stimulation.

Results: Comparing the factor of increase of migrated $\mathrm{mDC}$ counts due to mip $3 \mathrm{~b}$ stimulation, we detected a significant lower rate in samples of patients with arthritis (PsA) compared to those of patients without (Ps). Assays of mDCs without mip3b stimulation showed a significant higher count of migrated cells in the samples of the arthritic group [Figure 1]. Cell counts with Mip3b stimulation did vary slightly in the groups. The DC subpopulations and the expression of analyzed cell surface proteins did not show significant differences. The amounts of stimulated T-Lymphocytes did not differ significantly.
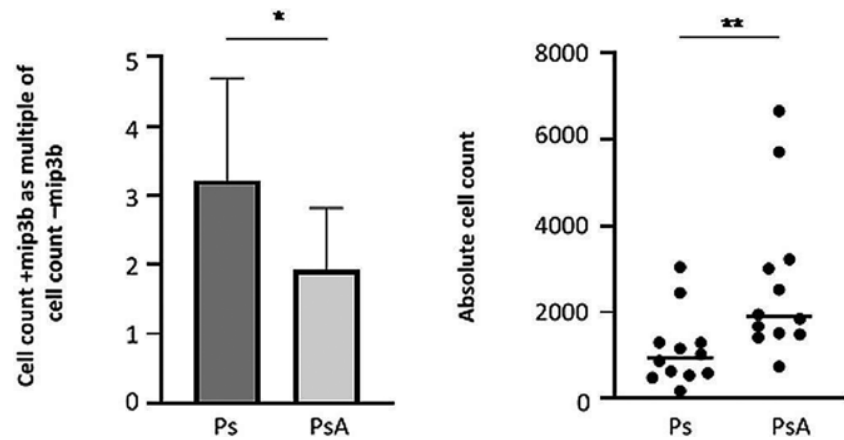

Figure 1. Migration essay showing mDCs following Mip3b (+miß3b) as multiples of mDCs without stimulation (-mip3b). The factor of increase is significantly lower in patients with arthritis (PsA) compared to patients without (Ps). Absolute counts of migrated mDCs without Mip3b are significantly higher in the arthritic group. Cell counts with stimulation do not differ significantly (data not shown). $\mathrm{N}=24, \mathrm{p}<0.05$

Conclusion: CCL19 (Mip3b) is a potent ligand to the CCR7 receptor inducing migration of DCs towards the lymphatic node [5]. The CCR7 amounts on the DC surface did not differ significantly in the groups. The mDCs without CCL19 stimulation migrated in higher amounts in samples of arthritic patients. Cell counts of stimulated DCs showed only slight differences. These results could be generated by a different appearance of the DCs of arthritic patients that might facilitate migration. Further experiments focusing on this aspect should be performed. A possible effect of disruptive factors (age, sex, medication...) needs to be clarified.

References:

[1] Henes, J.C., et al., High prevalence of psoriatic arthritis in dermatological patients with psoriasis: a cross-sectional study. Rheumatol Int, 2014. 34(2) p. 227-34.

[2] Lee, E.B., et al., Psoriasis risk factors and triggers. Cutis, 2018. 102(5s): $p$ 18-20.

[3] Kim, T.G., S.H. Kim, and M.G. Lee, The Origin of Skin Dendritic Cell Network and Its Role in Psoriasis. Int J Mol Sci, 2017. 19(1).

[4] Veale, D.J. and U. Fearon, The pathogenesis of psoriatic arthritis. Lancet 2018. 391(10136): p. 2273-2284.

[5] Ricart, B.G., et al., Dendritic cells distinguish individual chemokine signals through CCR7 and CXCR4. J Immunol, 2011. 186(1): p. 53-61.

Acknowledgments: This project was financially supported by Novartis Pharma $\mathrm{GmbH}$.

Disclosure of Interests: Sarah Schnitte Grant/research support from: Reaserch grant by Novartis, Alexander Fuchs: None declared, Tanja Funk: None declared 
Ann-Christin Pecher: None declared, Daniela Dörfel: None declared, Jörg Henes Grant/research support from: Novartis, Roche-Chugai, Consultant of: Novartis, Roche, Celgene, Pfizer, Abbvie, Sanofi, Boehringer-Ingelheim,

DOI: 10.1136/annrheumdis-2020-eular.4296

\begin{tabular}{|l|l}
\hline FRI0016 & R835, A NOVEL IRAK1/4 DUAL INHIBITOR IN CLINICAL \\
DEVELOPMENT, BLOCKS TOLL-LIKE RECEPTOR 4 \\
(TLR4) SIGNALING IN HUMAN AND MOUSE
\end{tabular}

C. Lamagna ${ }^{1}$, C. Gundel ${ }^{1}$, M. Chan ${ }^{1}$, C. Young ${ }^{1}$, S. Braselmann ${ }^{1}$, R. Frances ${ }^{1}$, S. Yi ${ }^{1}$, Y. Chen ${ }^{1}$, G. Park ${ }^{1}$, L. Chou ${ }^{1}$, E. Masuda ${ }^{1}$, V. Taylor ${ }^{1} .{ }^{1}$ Rigel Pharmaceuticals, South San Francisco, United States of America

Background: Toll-Like Receptors (TLR) and Interleukin-1 Receptors (IL-1R) play a critical role in the innate immune response as microbial sensors, providing a bridge between the innate and adaptive immunity (1). Interleukin receptor associated kinases (IRAK) 1 and 4 are serine/threonine kinases that are essential for signaling downstream of most TLRs and IL-1Rs and the resulting production of pro-inflammatory cytokines (2). Suppression of TLR and IL-1R signaling through inhibition of IRAK1/4 kinases is a promising therapeutic approach for the treatment of inflammatory and autoimmune diseases.

Objectives: The aim of the study was to characterize the effects of R835, a novel small molecule inhibitor of IRAK1/4, on TLR4 signaling.

Methods: R835 was identified in cell-based assays measuring cytokine production induced by LPS (TLR4 ligand). Inhibition of IRAK1 and IRAK4 kinases by R835 was confirmed in biochemical assays and cell lysates. The ability of R835 to inhibit TLR4 signaling was further evaluated in human and mouse whole blood assays. R835 was tested in a mouse model of LPS-induced cytokine release. Mice were pre-treated orally with vehicle or R835 prior to challenge with LPS and serum cytokine levels were monitored over a 24-hour period.

Results: We have identified R835, a selective small molecule inhibitor of IRAK1 and IRAK4. R835 blocked LPS/TLR4 signaling and the resulting production of proinflammatory cytokines in both human and mouse cells and whole blood. R835 suppressed serum cytokine elevation in mice challenged with LPS.

Conclusion: Our study demonstrates that R835, through inhibition of IRAK1/4 kinase activity, blocks LPS-induced cytokine production in vitro and in vivo. In a recent phase 1 study, R835 substantially reduced the increase of serum cytokines after an intravenous LPS challenge in healthy volunteers. Importantly, this shows that the pharmacological inhibition of IRAK1/4 pathway by R835 in humans mirrors the results obtained in mice. To our knowledge, R835 is the first dual IRAK1/4 inhibitor to enter clinical development and demonstrate inhibition of TLR4-induced cytokines in both mice and humans. R835 is a promising clinical candidate that will allow the exploration of IRAK1/4 inhibition in the treatment of cytokine-driven rheumatic and autoimmune diseases.

References:

[1] The interleukin-1 receptor/ Toll-like receptor superfamily: 10 years of progress. Luke A. J. O'Neill. Immunological Reviews 2008. Vol. 226: 10-18

[2] Flannery S, Bowie A G. The interleukin-1 receptor-associated kinases: Critical regulators of innate immune signaling. Biochemical Pharmacology, Volume 80, Issue 12, 15 December 2010, Pages 1981-1991.

Disclosure of Interests: Chrystelle Lamagna Shareholder of: Rigel Pharmaceuticals, Employee of: Rigel Pharmaceuticals, Caroline Gundel Shareholder of: Shareholder of Rigel Pharmaceuticals, Employee of: Employee of Rigel Pharmaceuticals, Meagan Chan Shareholder of: Rigel Pharmaceuticals,
Employee of: Rigel Pharmaceuticals, Chi Young Shareholder of: Rigel Pharmaceuticals, Employee of: Rigel Pharmaceuticals, Sylvia Braselmann Shareholder of: Shareholder of Rigel Pharmaceuticals, Employee of: Employee of Rigel Pharmaceuticals, Roy Frances Shareholder of: Rigel Pharmaceuticals, Employee of: Rigel Pharmaceuticals, Sothy Yi Shareholder of: Rigel Pharmaceuticals, Employee of: Rigel Pharmaceuticals, Yan Chen Shareholder of: Rigel Pharmaceuticals, Employee of: Rigel Pharmaceuticals, Gary Park Shareholder of: Rigel Pharmaceuticals, Employee of: Rigel Pharmaceuticals, Lu Chou Shareholder of: Rigel Pharmaceuticals, Employee of: Rigel Pharmaceuticals Esteban Masuda Shareholder of: Rigel Pharmaceuticals, Employee of: Rige Pharmaceuticals, Vanessa Taylor Shareholder of: Rigel Pharmaceuticals Employee of: Rigel Pharmaceuticals DOI: 10.1136/annrheumdis-2020-eular.3370

\section{FRI0017 \\ ACTIVATION OF TOLL-LIKE RECEPTORS IN PERIPHERAL BLOOD MONONUCLEAR CELLS OF TAKAYASU ARTERITIS PATIENTS}

Y. Tian ${ }^{1}$, J. Liं ${ }^{2}$, X. Tian ${ }^{2}$, X. Zeng ${ }^{2} .{ }^{1}$ Peking Union Medical College Hospital, Peking Union Medical College and Chinese Academy of Medical Sciences, National Clinical Research Center for Dermatologic and Immunologic Diseases (NCRC-DID), Rheumatology, Beijing, China; ${ }^{2}$ Peking Union Medical College Hospital, Peking Union Medical College and Chinese Academy of Medical Sciences, National Clinical Research Center for Dermatologic and Immunologic Diseases (NCRC-DID), Department of Rheumatology, Beijing, China

Background: The activation of self-specific T cells is essential in pathogenesis of Takayasu arteritis (TAK). Dendritic cell (DC) plays an indispensable role as the only antigen presenting cell for initial T cell, and Toll-like receptors (TLRs) are common source of activation signals for DCs. Then we speculate that there are activation of TLRs in TAK patients.

Objectives: To investigate the activation of TLRs in TAK patients.

Methods: Twenty-seven TAK patients were enrolled during April to October in 2019, with diagnosis met the 1990 criteria of American College of Rheumatology. Patient were divided into groups by the disease activity and medication history. Disease activity was assessed by the $1994 \mathrm{NIH}$ criteria. Quantitative Real-time Polymerase Chain Reaction (RT-qPCR) was used to analyze the mRNA relative abundance of 28 target genes in peripheral blood mononuclear cells (PBMCs). Differences between groups and correlation between any two genes were analyzed.

Results: The demographic data and clinical features of TAK patients were shown in Table 1. (1) Compared with health control (HC) group, mRNA abundance of TLR2, TLR4, P50, P65, IKBa, CTLA4, CD3, and BCL6 in untreated TAK group was upregulated $(<0.05)$, whereas mRNA abundance of $C D 40$ was downregulated ( $\mathrm{p}<0.05$ ). (2) Compared with HC group, mRNA abundance of TLR2, TLR4, IKBa $P D-1$ and $B C L 6$ in treated TAK group was upregulated $(p<0.05)$, whereas mRNA abundance of $L A G 3, C D 40$ and TCR was downregulated $(p<0.05)$. (3) Compared with untreated TAK group, mRNA abundance of P50, P65, CD28, CTLA4, TLR2, TLR4, IKBa, PD-1 and RORC was upregulated in treated TAK group ( $\mathrm{p}$ $<0.05)$. (4) Compared with non-active treated TAK group, mRNA abundance of p50, CD28, TCR, GATA3, RORC and FOXP3 was upregulated in nonactive treated TAK group $(\mathrm{p}<0.05)$. BCL6 showed correlation with the TLRs-NFKB pathway. (Figure 1 2, Table 2 )

Table 1. Demographic data and clinical features of patients with TAK

\begin{tabular}{|c|c|c|c|c|c|c|c|c|c|}
\hline & \multirow[b]{2}{*}{ Age (year) } & \multirow[b]{2}{*}{$\begin{array}{l}\text { Gender (male/ } \\
\text { female) }\end{array}$} & \multirow[b]{2}{*}{$\begin{array}{l}\text { Disease duration* } \\
\text { (months) }\end{array}$} & \multirow[b]{2}{*}{$\mathrm{ESR}(\mathrm{mm} / \mathrm{h})$} & \multirow[b]{2}{*}{ hs-CRP* (mg/L) } & \multirow[b]{2}{*}{$\begin{array}{l}\text { Interleukin } \\
6(\mathrm{pg} / \mathrm{mL})\end{array}$} & \multirow[b]{2}{*}{$\begin{array}{l}\text { TNFa(pg/ } \\
\mathrm{mL})\end{array}$} & \multicolumn{2}{|c|}{ Prednisone } \\
\hline & & & & & & & & $\begin{array}{c}\text { used/ } \\
\text { non-used }\end{array}$ & Dosage (mg/d) \\
\hline Treated $(n=20)$ & $39.37 \pm 9.27$ & $1 / 19$ & $43(12,103)$ & $14.60 \pm 8.94$ & $1(0.55,5.625)$ & $\begin{array}{l}2.1(2 \\
3.95)\end{array}$ & $7.56 \pm 4.39$ & $18 / 2$ & $10(10,32.5)$ \\
\hline Active $(n=11)$ & $39.30 \pm 7.889$ & $1 / 10$ & $118(16,166.5)$ & $16.82 \pm 10.81$ & $5.63(1.49,8.33)$ & $\begin{array}{l}3.15(2.025, \\
5.775)\end{array}$ & $8.42 \pm 5.57$ & $10 / 1$ & $10(10,15)$ \\
\hline Nonactive $(n=9)$ & $39.44 \pm 10.59$ & $0 / 9$ & $40(12,44)$ & $11.89 \pm 4.61$ & $0.84(0.31,1)$ & $2(2,2.4)$ & $6.60 \pm 2.11$ & $8 / 1$ & $8.75(6.875,16.25)$ \\
\hline$P$ value & 0.89 & - & 0.16 & 0.34 & 0.02 & 0.08 & 0.65 & - & 0.37 \\
\hline \multicolumn{10}{|l|}{ Untreated $(n=7)$} \\
\hline \multicolumn{10}{|l|}{ Active $(n=4)$} \\
\hline 1 & 31 & $M$ & - & 91 & 140.72 & - & - & - & 0 \\
\hline 2 & 25 & $\mathrm{~F}$ & - & 19 & 11.28 & 6.3 & 5.2 & - & 0 \\
\hline 3 & 23 & M & - & 71 & 77.36 & 6.3 & 6.2 & - & 0 \\
\hline 4 & 29 & $\mathrm{~F}$ & - & 127 & 113.62 & 22.2 & 8.4 & - & 0 \\
\hline \multicolumn{10}{|l|}{ Nonactive $(n=3)$} \\
\hline 5 & 34 & $F$ & - & 7 & 0.34 & 2 & 4.3 & - & 0 \\
\hline 6 & 27 & $\mathrm{~F}$ & - & 14 & 0.16 & 25.7 & 4 & - & 0 \\
\hline 7 & 38 & $\mathrm{~F}$ & - & 5 & 0.32 & 3 & 4 & - & 0 \\
\hline
\end{tabular}

* median (min, max) 